\title{
Sleep symptoms and polysomnographic architecture in advanced Parkinson's disease after chronic bilateral subthalamic stimulation
}

\author{
A Iranzo, F Valldeoriola, J Santamaría, E Tolosa, J Rumià
}

J Neurol Neurosurg Psychiatry 2002;72:661-664

\begin{abstract}
Objective: To evaluate the sleep symptoms and polysomnographic architecture in advanced Parkinson's disease after chronic bilateral subthalamic stimulation (STN-DBS). Methods: Sleep was studied in 11 patients (six women and five men; mean (SD) age 63.6 (7.8) years) who underwent STN-DBS. Subjective sleep evaluation was assessed by clinical sleep interview and the Pittsburgh sleep quality index (PSQI) questionnaire, and sleep architecture by polysomnography with audiovisual recording. Nocturnal mobility was evaluated.

Results: Before surgery, eight patients rated their sleep quality as unsatisfactory; seven of these had a marked improvement after surgery, and the PSQI questionnaire showed significantly improved sleep quality. After surgery, polysomnography showed an increase in the longest period of uninterrupted sleep and a decrease in the arousal index. There was an increase in nocturnal mobility after surgery, but no change in REM sleep behaviour disorder.

Conclusions: In advanced Parkinson's disease, chronic STN-DBS is associated with subjective improvement in sleep quality, probably through increased nocturnal mobility and reduction of sleep fragmentation.
\end{abstract}

1

n advanced Parkinson's disease, sleep disturbances are common, and polysomnographic recordings have shown disrupted sleep architecture, rapid eye movement (REM) sleep behaviour disorder, and periodic limb movements during sleep. These alterations have been related to several conditions such as nocturnal rigidity, hypokinesia, pain, the effects of antiparkinsonian drugs on sleep, coexisting anxiety and depression, and dysfunction of the brain structures involved in sleep regulation.

In Parkinson's disease, chronic bilateral deep brain stimulation of the subthalamic nucleus (STN-DBS) improves the parkinsonian symptoms and in most cases reduces the need for dopaminergic drugs. ${ }^{23}$ In our centre, some patients treated with STN-DBS have reported a marked improvement of their sleep quality after surgery. The objective of our study was to assess the sleep symptoms and polysomnographic architecture in a group of patients with advanced Parkinson's disease after chronic bilateral STN-DBS.

\section{METHODS}

We evaluated prospectively 11 consecutive patients with advanced Parkinson's disease submitted to STN-DBS. The evaluations were made during the week preceding surgery and again six months later. Patient selection criteria and the stereotactic surgical procedures have been described in a previous paper. ${ }^{3}$ After surgery, antiparkinsonian drugs were tapered and the electrical stimulation parameters were set to obtain the best clinical results. All patients were instructed to keep the stimulation turned on throughout the day and night. During the six months of the study period, benzodiazepines and antidepressants remained unchanged in those patients who had been taking these drugs chronically before surgery.

\section{Subjective sleep evaluation}

Evaluations included a complete sleep history and the Pittsburgh sleep quality index (PSQI) questionnaire. ${ }^{4}$ Excessive daytime sleepiness was estimated by the Epworth sleepiness scale. ${ }^{5}$

\section{Polysomnographic evaluation}

Patients underwent polysomnographic studies with continuous audiovisual recording on two consecutive nights both before and after surgery. The first preoperative and postoperative nights were considered to be adaptation nights and no analyses were done on those nights. Although the patients knew that surgery was planned several days after preoperative polysomnographic studies, they all reported that the nights they spent in the sleep laboratory were comparable with their habitual sleep at home.

Polysomnographic recordings were made digitally by the standard method. To avoid the marked artefact on the EEG channels produced by the stimulation devices, instead of referencing $\mathrm{C} 3, \mathrm{C} 4, \mathrm{O} 1$, and $\mathrm{O} 2$ to the ears, we used the following montage: $\mathrm{F} 3$ to $\mathrm{C} 4, \mathrm{~F} 4$ to $\mathrm{C} 3$, and $\mathrm{O} 1$ to $\mathrm{O} 2$. We also had to decrease the high frequency filter to $15 \mathrm{~Hz}$ in the EEG channels and to $35 \mathrm{~Hz}$ in the electromyographic channels. Sleep recordings obtained with this montage allowed sleep stage identification, which otherwise followed standard criteria. ${ }^{6}$ Spindles appearing in one or more of the EEG channels were counted visually during two separate 10 minute epochs of stage II, and during two non-consecutive 10 minute epochs of stages III and IV. The phasic electromyographic density during REM sleep was calculated according to the method described by Lapierre and Montplaisir. ${ }^{7}$

A history of restless sleep associated with nightmares plus detection of vigorous movements associated with excessive phasic and tonic electromyographic activity during REM sleep by videomonitoring established the diagnosis of REM sleep behaviour disorder. Periodic limb movements during sleep were determined by standard criteria. ${ }^{8}$ Videotapes synchronised with the polysomnographic recordings were reviewed to check the numbers of body position changes and abnormal movements.

\section{Parkinsonism and psychopathological evaluation}

To assess the antiparkinsonian effect of STN-DBS, we followed the instructions given by the CAPIT protocol. ${ }^{9}$ Nocturnal mobility was evaluated by item 12 of the UPDRS-II ("turning in bed and adjusting bed clothes"). Psychopathological status 
Table 1 Results of the components and the global score of the Pittsburgh sleep quality index (PSQI) questionnaire before and six months after surgery $(n=11)$

\begin{tabular}{|c|c|c|c|}
\hline & Before surgery & After surgery & $p$ Value \\
\hline Subjective sleep quality & $2.5(0.6)$ & $0.4(0.6)$ & $<0.001 *$ \\
\hline Sleep latency & 2.0 (1.3) & $0.4(0.9)$ & $0.002 *$ \\
\hline Sleep duration & $2.6(0.6)$ & $0.7(0.6)$ & $<0.001$ * \\
\hline Habitual sleep efficiency & $2.4(0.8)$ & 0.5 (1.0) & $0.001 *$ \\
\hline Sleep disturbances & $1.7(0.4)$ & $0.9(0.5)$ & $0.005^{*}$ \\
\hline Use of drugs for sleep & $1.9(1.5)$ & $1.9(1.5)$ & 1 \\
\hline Daytime dysfunction & $1.5(1.0)$ & $0.6(0.6)$ & $0.005^{*}$ \\
\hline PSQI global score & $14.8(4.5)$ & $5.4(4.6)$ & $<0.001^{*}$ \\
\hline \multicolumn{4}{|c|}{$\begin{array}{l}\text { Results are mean (SD). } \\
\text { Each component of the PSQI ranges from } 0 \text { (no impairment) to } 3 \text { (maximum impairment). The sum of the } \\
\text { scores of the seven components yields the global PSQI score, which has a range of } 0-21 \text {; higer scores } \\
\text { indicate worse sleep quality. } \\
{ }^{*} p<0.05 \text {. }\end{array}$} \\
\hline
\end{tabular}

was evaluated by the hospital anxiety and depression scale. ${ }^{10}$ Total levodopa equivalent dose was calculated as in a previous report. ${ }^{11}$

\section{Statistical analysis}

Values are given as mean (SD). Comparisons before and after surgery for sleep measures, parkinsonian symptoms, and psychopathological status were done by the Student's $t$ test or the Wilcoxon signed rank test as appropriate.

\section{RESULTS}

We studied six women and five men with advanced Parkinson's disease with a mean age of 63.6 (7.8) years, mean disease duration 17.3 (9.1) years, mean presurgical off medication Hoehn and Yahr stage 4.2 (0.8), UPDRS-III (total motor score) 54.8 (12), and UPDRS-II (activities of daily living) 29.9 (7.9).

\section{Subjective sleep evaluation}

Before surgery, eight patients rated their sleep quality as unsatisfactory, and marked improvement was reported by seven of these after surgery. Postoperatively, satisfactory sleep was reported by 10 patients, difficulty with falling asleep resolved in five of seven, frequent awakenings in seven of eight, and early awakening in one of two. The PSQI questionnaire showed a significant improvement in sleep quality, a decrease in sleep latency, sleep disturbances and daytime dysfunction, and an increase in sleep duration and habitual sleep efficiency (table 1).

In eight patients-four men and four women-nocturnal vigorous movements and vocalisations were reported, accompanied by the recall of nightmares both before and after surgery. Habitual snoring was present in four patients before and after surgery. Before surgery, only one patient had mild daytime sleepiness (Epworth sleepiness scale score of 10/21), which did not change after surgery.

None of the patients reported restless legs, early morning dystonia, nocturnal akathisia, or diurnal sleep attacks before and after surgery.

\section{Polysomnographic evaluation}

The polysomnographic findings are reported in table 2. After surgery, there was a significant increase in the number of changes of body position and in the longest period of uninterrupted sleep, and a decrease in the arousal index. Although there was a trend towards an increase in the percentage of stage III-IV, and a decrease in stage II and in the number of awakenings, these differences did not reach significance. There were no other significant differences between the preoperative and postoperative polysomnographs.

Before surgery, REM sleep behaviour disorder was detected by polysomnography in the same eight patients who reported a history of vigorous movements during sleep associated with

Table 2 Polysomnographic measures before and after surgery

\begin{tabular}{llll} 
& Before surgery & After surgery & p Value \\
\hline Changes of body position (n) & $0.9(1.3)$ & $3.7(3.6)$ & $0.04^{*}$ \\
Time in bed (min) & $405.8(120.2)$ & $447.1(20.6)$ & 0.33 \\
Total sleep time (min) & $303.6(64.0)$ & $253.4(115.6)$ & 0.15 \\
Sleep efficiency (\%) & $68.6(12.6)$ & $62.5(17.6)$ & 0.24 \\
Sleep onset latency (min) & $44(59)$ & $21.4(19.7)$ & 0.56 \\
Longest continuous sleep period (min) & $33.4(15.5)$ & $51.3(33.5)$ & $0.02^{*}$ \\
Wake time after sleep onset (min) & $110.6(54.6)$ & $98.8(78.8)$ & 0.47 \\
Awakenings (n) & $17.6(12)$ & $12.8(10.5)$ & 0.05 \\
Arousal index & $18.0(11.3)$ & $11.2(12.4)$ & $0.02^{*}$ \\
Stage I (\%) & $10.1(10.2)$ & $12.3(11.7)$ & 0.42 \\
Stage II (\%) & $54.9(16.8)$ & $45.7(18.8)$ & 0.07 \\
Stage III-IV (\%) & $15.5(9.6)$ & $22.2(11.2)$ & 0.05 \\
REM (\%) & $19.4(9.8)$ & $19.6(12.1)$ & 0.92 \\
Phasic REM submental density (\%) & $38(63.6)$ & $17.4(15.6)$ & 0.54 \\
PLMS index & $6.8(9.7)$ & $13.7(16.5)$ & 0.20 \\
Spindles stage II (+) & $35.2(30.0)$ & $31.1(24.0)$ & 0.23 \\
Spindles stage III-IV (+) & $16.0(14.0)$ & $22.2(30.3)$ & 0.62 \\
Apnoea-hypopnoea index & $4.2(3.9)$ & $4.8(4.3)$ & 0.81 \\
\hline Values are mean (SD). & & & \\
* p<O.05. & & & \\
PLMS, periodic limb movement during sleep; REM, rapid eye movements; (+), total number of spindles per 20 \\
minutes.
\end{tabular}


nightmares. After surgery, REM sleep behaviour disorder was still present in these eight subjects as polysomnography continued to detect excessive REM sleep muscle twitching associated with vigorous movements. There were no significant differences between phasic REM submental density before and after surgery.

The mean index of periodic limb movements during sleep (PLMS index; the number of periodic leg movements per hour of sleep) increased after surgery, but the difference was not significant. Before surgery, the PLMS index was greater than 10 in two subjects; after surgery, this index was greater than 10 in these two patients plus three others.

Although 10 patients gained weight after surgery, none showed an apnoea-hypopnoea index (number of apnoeas/ hypopnoeas per hour of sleep) greater than 10.

\section{Parkinsonism and psychopathological evaluation}

The UPDRS-III improved by $63.5 \%$ ( $\mathrm{p}<0.001)$, UPDRS-II by $64.3 \%(\mathrm{p}<0.001)$, and Hoehn and Yahr changed from $4.2(0.8)$ to $2.6(0.5)(\mathrm{p}<0.001)$ when the off-medication state before surgery was compared with the off-medication/on-stimulation state after surgery. Nocturnal mobility (UPDRS-II, item 12) improved significantly from 2.4 (1.3) before surgery to $0.8(0.7)$ after surgery $(\mathrm{p}=0.006)$. The "off" time was reduced by $80.3 \%$ and the intensity of dyskinesias by $88.7 \%(\mathrm{p}<0.001)$.

After surgery, there was a significant improvement in the hospital anxiety and depression scale subscores for anxiety (7.93 (3.90) $v 3.93(4.31), \mathrm{p}<0.001)$ and depression $(7.93$ (4.18) v $3.56(4.42), \mathrm{p}=0.006)$.

Before surgery, all patients were taking levodopa and nine were taking dopamine agonists (ropinirole in five, pergolide in two, bromocriptine in one, and ropinirole plus subcutaneous apomorphine in one). Six months after surgery, dopamine agonists had been withdrawn in all patients, and two were not taking any dopaminergic medication. The remaining nine patients were taking only levodopa, with a mean dose reduction of $65 \%$. In all patients, both before and after surgery, the last dose of the dopaminergic agents was taken at bedtime. Seven subjects were being treated chronically with hypnotics (lorazepam in four, zolpidem in two, and flunitrazepam in one), and six with antidepressants (paroxetine in two, fluoxetine in one, amitriptyline in two, and imipramine in one). None of the patients was taking clonazepam or antipsychotic agents.

\section{DISCUSSION}

In this study in patients with advanced Parkinson's disease, sleep symptoms and nocturnal mobility improved six months after the onset of STN-DBS when compared with preoperative evaluations. Polysomnographic recordings after surgery showed more continuous sleep but persistence of REM sleep behaviour disorder and periodic limb movements during sleep. When analysing such results, all motor, mood, and pharmacological changes associated with the surgical procedure during the time following surgery should be considered. For example, dopaminergic drugs were reduced after surgery, and this change could have modified the postoperative sleep architecture. ${ }^{1}$ Dopaminergic drug reduction is part of the current medical strategy that accompanies STN-DBS. ${ }^{3}$

This study has some limitations. Considering the many variables that influence sleep in patients with Parkinson's disease, the number of patients evaluated is relatively small, and we have not performed comparative studies in patients undergoing other neurosurgical procedures such as pallidotomy, or in those receiving only medical treatment. However, it can be assumed that in subjects being treated only with drugs, sleep quality is unlikely to improve with the passage of time and worsening of the disease. Another limitation stems from the fact that polysomnographic studies could not be scored blind because they were easily recognised as post-surgical by the artefacts produced by continuous electrical stimulation.
Before surgery, most of the patients rated their sleep quality as unsatisfactory, and polysomnography detected fragmented sleep and the frequent presence of REM sleep behaviour disorder and periodic limb movements during sleep. Six months postoperatively, the PSQI questionnaire showed a marked improvement in sleep quality, and polysomnographic recordings showed an increase in the number of body position changes, an increase in the longest period of uninterrupted sleep, and a decrease in the arousal index. The patients' subjective improvement in sleep quality might be explained in part by the amelioration in nocturnal mobility and by a more continuous sleep pattern, but a reduction in dopaminergic drug treatment, an improvement in functional disability and in psychopathological status, or other factors could have contributed to the improvement.

The persistence of REM sleep behaviour disorder and periodic limb movements during sleep after surgery indicates that electrical deactivation of the subthalamic nucleus does not eliminate these types of abnormal nocturnal motor activity. REM sleep behaviour disorder is thought to result mainly from a dysfunction of the pedunculopontine nucleus, which is a structure that has strong anatomical connections with the subthalamic nucleus and the substantia nigra, ${ }^{12}$ and is functionally impaired in Parkinson's disease. ${ }^{13}$ Thus the persistence of REM sleep behaviour disorder after surgery suggests that stimulation of the subthalamic nucleus does not restore the pedunculopontine activity which promotes muscle atonia during REM sleep.

After surgery there was an increase in the mean PLMS index, and in three subjects an index greater than 10 was only detected after the surgical procedure. This increase in periodic limb movements might reflect the reduction in postoperative dopaminergic drug treatment, as the PLMS index is significantly higher in patients with Parkinson's disease who are off drugs than in healthy age matched controls, ${ }^{14}$ and is decreased by dopaminergic drugs. ${ }^{15}$

Recently, Arnulf et al evaluated the postoperative polysomnographic records of 10 parkinsonian patients treated for three to six months with STN-DBS in "off" and "on" conditions, separated by a two day interval. ${ }^{16}$ Their results differed from ours in that during STN-DBS the total sleep time was increased and awake time after sleep onset was decreased. The sudden discontinuation of chronic STN-DBS in their patients could have caused reduced and fragmented sleep owing to the unavoidable worsening of their motor state, and this might have influenced their results. It has been our experience that abrupt discontinuation of STN stimulation during the day results consistently in an aggravation of the patient's neurological condition.

\section{Conclusions}

In advanced Parkinson's disease, chronic bilateral subthalamic stimulation is associated with subjective sleep improvement, probably resulting from amelioration of nocturnal mobility and reduction of sleep fragmentation. STN-DBS does not eliminate REM sleep behaviour disorder and periodic limb movements during sleep.

\section{Authors' affiliations}

A Iranzo, F Valldeoriola, J Santamaría, E Tolosa, Service of

Neurology, Hospital Clinic i Provincial de Barcelona, Barcelona, Spain

J Rumià, Service of Neurosurgery, Hospital Clinic i Provincial de Barcelona

Correspondence to: Dr Alex Iranzo, Neurology Service, Hospital Clinic de Barcelona, C/Villarroel 170, Barcelona 08036, Spain;

airanzo@clinic.ub.es

Received 9 October 2001

In revised form 4 January 2002

Accepted 10 January 2002 


\section{REFERENCES}

1 Aldrich MS. Parkinson's disease and sleep disturbance. In: Aldrich MS, ed. Sleep medicine. New York: Oxford University Press, 1999:329-32.

2 Deep-brain Stimulation for Parkinson's Disease Study Group. Deep-brain stimulation of the subthalamic nucleus or the pars interna of the globus pallidus in Parkinson's disease. N Engl J Med 2001;345:956-63.

3 Molinuevo JL, Valldeoriola F, Tolosa E, et al. Levodopa withdrawal after bilateral subthalamic nucleus stimulation in advanced Parkinson disease. Arch Neurol 2000;57;983-8

4 Buysse DJ, Reynolds CF, Monk TH, et al. The Pittsburgh sleep quality index: a new instrument for psychiatric practice and research. Psychiatry Res 1989:28:193-213.

5 Johns MW. A new method for measuring daytime sleepiness: the Epworth sleepiness scale. Sleep 1991;14:540-5.

6 Rechtschaffen A, Kales A. A manual of standardized terminology, techniques and scoring system for sleep stages of human subjects. Los Angeles: Brain Information Service, Brain Research Institute, 1968.

7 Lapierre O, Montplaisir J. Polysomnographic features of REM sleep behavior disorder: development of a scoring method. Neurology 1992;42:1371-4.
8 Guilleminault C (chairman), Atlas Task Force of the American Sleep Disorders Association. Recording and scoring leg movements. Sleep 1993; 16:748-59.

9 Langston JW, Widner H, Goetz CG, et al. Core assessment program for intracerebral transplantations (CAPIT). Mov Disord 1992;7:2-13.

10 Zigmond AS, Snaith RP. The hospital anxiety and depression scale. Acta Psychiatr Scand 1983:67:361-70.

11 Baron MS, Vitek JL, Bakay RAE, et al. Treatment of advanced Parkinson's disease by posterior GPi pallidotomy: 1-year results of a pilot study. Ann Neurol 1996;40:355-66

12 Rye DB. Contributions of the pedunculopontine region to normal and altered REM sleep. Sleep 1997:20:757-88.

13 Pahapill PA, Lozano AM. The pedunculopontine nucleus and Parkinson's disease. Brain 2000;1 23:1767-83.

14 Wetter TC, Collado-Seidel V, Polmächer T, et al. Sleep and periodic leg movement patterns in drug-free patients with Parkinson's disease and multiple system atrophy. Sleep 2000;23:361-6.

15 Hening W, Allen R, Early C, et al. The treatment of restless legs syndrome and periodic limb movement disorder. Sleep 1999:22:970-99.

16 Arnulf I, Bejiani BP, Garma L, et al. Improvement of sleep architecture in PD with subthalamic stimulation. Neurology 2000;55:1732-4.

\section{NEUROLOGICAL STAMP}

\section{Josef Brudzinski (1874-1917)}

oseph Brudzinski was a Polish physician who graduated from Moscow in 1897 and specialised in paediatrics. He subsequently became interested in abnormal reflexes associated with meningitis. After performing animal experiments he came to the conclusion that the neurological signs in meningitis were due to irritation of the posterior nerve roots, changes in intracranial pressure, alteration of the circulation within the brain and increased muscle tone. He is remembered eponymically for Brudinzki's signs, of which there are four; his "nape of the neck" sign is best known. No studies subsequent to his original report have evaluated Brudinski's signs. Most of the signs were described in patients who had tuberculous meningitis.

Philatelically a postcard issued by Poland in 1970 honoured him.

L F Haas

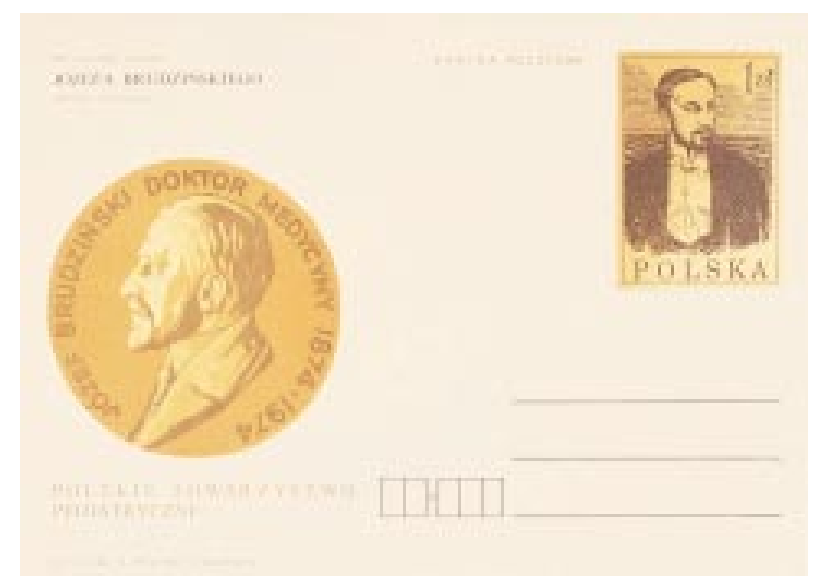

